\title{
On asymptotic cyclicity of doubly stochastic operators
}

\author{
by Wojciech Bartoszek (Pretoria)
}

\begin{abstract}
It is proved that a doubly stochastic operator $P$ is weakly asymptotically cyclic if it almost overlaps supports. If moreover $P$ is Frobenius-Perron or Harris then it is strongly asymptotically cyclic.
\end{abstract}

1. Introduction. Let $(X, \mathcal{A}, \mu)$ be a (complete) $\sigma$-finite measure space. The Banach lattice of real $\mathcal{A}$-measurable functions $f$ such that $|f|^{p}$ is $\mu$ integrable (resp. ess sup $|f|<\infty$ ) is denoted by $L^{p}(\mu)$ (resp. $L^{\infty}(\mu)$ ). $\|\cdot\|_{p}$ stands for the relevant norm. Functions equal $\mu$-almost everywhere are identified. A linear operator $P: L^{1}(\mu) \rightarrow L^{1}(\mu)$ is called Markov if $P f \geq 0$ and $\|P f\|_{1}=\|f\|_{1}$ for all $f \geq 0, f \in L^{1}(\mu)$. By $\mathcal{D}=\mathcal{D}(X, \mathcal{A}, \mu)$ we denote the set of all (normalized) densities on $X$, that is,

$$
\mathcal{D}=\left\{f \in L^{1}(\mu): f \geq 0,\|f\|_{1}=1\right\} .
$$

We say that $f_{*} \in \mathcal{D}$ is stationary if $P f_{*}=f_{*}$. If $(X, \mathcal{A}, \mu)$ is a probability space and $P \mathbf{1}=\mathbf{1}$ then a Markov operator $P$ is called doubly stochastic (or doubly markovian). An important basic property of doubly stochastic operators is that together with their adjoints, they are positive linear contractions on each $L^{p}(\mu)$, where $1 \leq p \leq \infty$ (see Proposition 1.1 in [Br] for the details). In particular, instead of studying the convergence on $L^{1}$ we may pass to $L^{2}$ if necessary. It is a routine trick to identify a Markov operator $P$ possessing a stationary, strictly positive density $f_{*}$ with its rescaled version $\bar{P} f=P\left(f f_{*}\right) / f_{*}$, which is defined on $L^{1}\left(f_{*} d \mu\right)$. Clearly $\bar{P}$ is doubly stochastic. Therefore our results are formulated only for doubly stochastic operators. Their generalizations to Markov operators with strictly positive

1991 Mathematics Subject Classification: Primary 47A35.

Key words and phrases: doubly stochastic operator, asymptotic stability, mixing.

This paper has been completed during the sabbatical visit to the Department of Mathematics, Wrocław University of Technology in July-September 1998. The author wishes to thank the University of South Africa for the sabbatical leave. Financial support from the Foundation for Research Development and UNISA Research Fund is gratefully acknowledged. 
stationary densities are obvious. The strict positivity assumption may be removed if $P$ is a Frobenius-Perron operator (see [Z]).

Let $S: X \rightarrow X$ be a nonsingular (i.e. $\mu\left(S^{-1}(A)\right)=0$ if $\mu(A)=0$ ) measurable transformation of $(X, \mathcal{A}, \mu)$. Recall that the corresponding Frobenius-Perron operator $P_{S}: L^{1}(\mu) \rightarrow L^{1}(\mu)$ is defined by $\int_{A} P_{S} f d \mu=$ $\int_{S^{-1}(A)} f d \mu$. Clearly $P_{S}$ is a Markov operator and its adjoint $P_{S}^{*}: L^{\infty}(\mu) \rightarrow$ $L^{\infty}(\mu)$ is the composition operator $P_{S}^{*} h=h \circ S$ and is called the Koopman operator. If $S$ preserves $\mu$ then the Koopman operator $P_{S}^{*}$ is isometric on each $L^{p}(\mu)$, noninvertible in general.

Definition 1. A Markov operator $P: L^{1}(\mu) \rightarrow L^{1}(\mu)$ is called strongly asymptotically cyclic if there exist a finite family of densities $g_{1}, \ldots, g_{r}$ and linear functionals $\Lambda_{1}, \ldots, \Lambda_{r}$ such that

$$
\lim _{n \rightarrow \infty}\left\|P^{n} f-\sum_{j=1}^{r} \Lambda_{j}(f) g_{(j+n) \bmod r}\right\|_{1}=0
$$

for all $f \in \mathcal{D}$. If $r=1$ then $P$ is called asymptotically stable. We also say that $P$ is weakly asymptotically cyclic (w.a.c.) if the convergence (1) holds for the weak topology only.

Asymptotic properties of iterates of doubly stochastic operators have been extensively studied (see [B1], [B2], [B3], [BB], [K1], [K2], [R1], [R2], $[\mathrm{Z}])$. For a comprehensive review of the subject and many examples the reader is referred to the monograph $[\mathrm{LM}]$.

It has been proved in [BB] (see also [R1]) that if $P$ is Harris or FrobeniusPerron then asymptotic stability holds whenever $P$ overlaps supports (i.e. $P^{n} f_{1} \wedge P^{n} f_{2} \neq 0$ for all densities $f_{1}, f_{2}$ and $n$ large enough). In this paper the concept of overlapping is generalized. We discuss how asymptotic properties of iterates are affected. We introduce the following:

Definition 2. We say that a Markov operator $P: L^{1}(\mu) \rightarrow L^{1}(\mu)$ almost overlaps supports (a.o.s. for abbreviation) if there exists $d \geq 0$ such that for all densities $f_{1}, f_{2} \in \mathcal{D}$ there exist $n=n\left(f_{1}, f_{2}\right)$ and $m=m\left(f_{1}, f_{2}\right)$ such that $|n-m| \leq d$ and $P^{n} f_{1} \wedge P^{m} f_{2} \neq 0$, where $\wedge$ stands for the ordinary minimum in $L^{1}(\mu)$.

Definition 3. We say that a Markov operator $P: L^{1}(\mu) \rightarrow L^{1}(\mu)$ individually almost overlaps supports (i.a.o.s. for abbreviation) if there exists $d \geq 1$ such that for every density $f \in \mathcal{D}$ there exist $n=n(f)<m=m(f) \leq$ $n+d$ such that $P^{n} f \wedge P^{m} f \neq 0$.

If for every $f \in L^{1}(X, \mathcal{A}, \mu)$ the iterates $P^{n} f$ have a norm convergent subsequence (i.e. $\omega_{1}(f)=\left\{g:\left\|P^{n_{k}} f-g\right\|_{1} \rightarrow 0\right.$ for some $\left.n_{k} \rightarrow \infty\right\} \neq \emptyset$ ) and if $P$ a.o.s. then $P$ is asymptotically cyclic (see [B3]). Similar results were obtained in [B1] for kernel Markov operators (i.e. $\operatorname{Pf}(x)=\int k(x, y) f(y) d \mu(y)$ 
for suitable $k(x, y))$. In this case $\omega_{1}(f) \neq \emptyset$ is compact due to Krasnosel'skiı's theorem (see $[\mathrm{L}]$ for a self-contained proof). Our current approach differs from $[\mathrm{BB}]$ and is based on ideas of $[\mathrm{F}]$, where most of our notation and terminology come from. We briefly recall the necessary ones. A Markov operator $P: L^{1}(\mu) \rightarrow L^{1}(\mu)$ is said to be conservative if for some (equivalently, all) strictly positive $f \in L^{1}(\mu)$ we have $\sum_{n=0}^{\infty} P^{n} f(x)=\infty \mu$-a.e. It is well known that if $P^{*} h \leq h$ for some $h \in L^{\infty}(\mu)$ then $P^{*} h=h$ whenever $P$ is conservative. Clearly each Markov operator with strictly positive stationary density is conservative. Let us recall that conservative Markov operators $P$ (in particular all doubly stochastic operators) are nondisappearing, i.e. if $P^{*} f=0$ for some $f \geq 0$ then $f=0$. Hence (see Lemma 0 in [KL] for the details) if $P^{*} g=\mathbf{1}_{A}$ with $0 \leq g \leq 1$ then there exists a unique $E \in \mathcal{A}$ such that $g=\mathbf{1}_{E}$. The family of all $A \in \mathcal{A}$ such that for every $n$ there exists $A_{n} \in \mathcal{A}$ such that $P^{* n} \mathbf{1}_{A}=\mathbf{1}_{A_{n}}$ is denoted by $\Sigma_{\mathrm{d}}(P)$. Clearly $\Sigma_{\mathrm{d}}(P)$ is a sub- $\sigma$-algebra if $P$ is doubly stochastic, and it is then called a deterministic $\sigma$-algebra. By $\Sigma_{1}(P)$ we denote the sub- $\sigma$-algebra of $\Sigma_{\mathrm{d}}(P)$ consisting of all $A$ such that for every natural $n$ we have $P^{* n} P^{n} \mathbf{1}_{A}=P^{n} P^{* n} \mathbf{1}_{A}=\mathbf{1}_{A}$ (see $[\mathrm{F}]$ for the details). By symmetry $\Sigma_{1}(P)=\Sigma_{1}\left(P^{*}\right)$.

We start with the following:

Proposition 1. Let $P$ be a doubly stochastic operator on $L^{1}(X, \mathcal{A}, \mu)$. If $P$ i.a.o.s. then there exists $r \leq d$ ! such that $P^{r} \mathbf{1}_{A}=\mathbf{1}_{A}$ for all $A \in$ $\Sigma_{\mathrm{d}}\left(P^{*}\right)=\Sigma_{1}(P)$, where $d$ comes from Definition 3. Moreover for every $f \in L^{p}(X, \mathcal{A}, \mu)$, weak $\lim _{n \rightarrow \infty} P^{r n} f$ exists and belongs to $L^{p}\left(X, \Sigma_{\mathrm{d}}\left(P^{*}\right), \mu\right)$. If $P$ a.o.s. then $\Sigma_{\mathrm{d}}\left(P^{*}\right)=\Sigma_{1}(P)$ is finite (atomic) and consists of at most $d+1$ atoms.

Proof. Given $A \in \Sigma_{\mathrm{d}}\left(P^{*}\right)$ we consider the maximal natural $r_{A}$ for which there exists $\Sigma_{\mathrm{d}}\left(P^{*}\right) \ni B \subseteq A$ such that

$$
\mathbf{1}_{B}, P \mathbf{1}_{B}=\mathbf{1}_{B_{1}}, \ldots, P^{r-1} \mathbf{1}_{B}=\mathbf{1}_{B_{r-1}}
$$

are pairwise orthogonal. We notice that always $r_{A} \leq d$. In fact, by the i.a.o.s. assumption we can choose $n<m$ with $m-n \leq d$ such that $P^{m} \mathbf{1}_{B} \wedge P^{n} \mathbf{1}_{B}$ $\neq 0$. Then

$$
0 \neq P^{* n} P^{m} \mathbf{1}_{B} \wedge P^{* n} P^{n} \mathbf{1}_{B}=P^{m-n} \mathbf{1}_{B} \wedge \mathbf{1}_{B},
$$

and $r_{A} \leq d$ follows.

If $P^{r_{A}} \mathbf{1}_{B} \neq \mathbf{1}_{B}$ then we define $D=B \backslash B_{r_{A}} \neq \emptyset$. Clearly $D \subseteq A$ and

$$
\mathbf{1}_{D}, P \mathbf{1}_{D}=\mathbf{1}_{D_{1}}, \ldots, P^{r_{A}} \mathbf{1}_{D}=\mathbf{1}_{D_{r_{A}}}
$$

are pairwise orthogonal, contradicting the maximality of $r_{A}$. Hence $P^{r_{A}} \mathbf{1}_{B}$ $=\mathbf{1}_{B}$.

Now let

$$
\mathcal{C}_{A}=\left\{B \in \Sigma_{\mathrm{d}}\left(P^{*}\right): B \subseteq A \text {, and } P^{r} \mathbf{1}_{B}=\mathbf{1}_{B} \text { for some } 1 \leq r \leq d\right\} .
$$


It is not hard to see that $A=\bigcup_{j=1}^{d} B_{j}$, where $P^{j} \mathbf{1}_{B_{j}}=\mathbf{1}_{B_{j}}$ for every $j$ (some $B_{j}$ may be empty). Finally, define $R_{A}=\operatorname{LCM}\left\{j: B_{j} \neq\right.$ $\emptyset\} \leq d$ !. Then $P^{R_{A}} \mathbf{1}_{B}=\mathbf{1}_{B}$ for every $B \in \Sigma_{\mathrm{d}}\left(P^{*}\right) \cap A$. Substituting $X=A$ we get $P^{r} \mathbf{1}_{B}=\mathbf{1}_{B}$ for all $B \in \Sigma_{\mathrm{d}}\left(P^{*}\right)$, where $r=R_{X}$. In particular $P^{r}=$ Id on $L^{p}\left(X, \Sigma_{\mathrm{d}}\left(P^{*}\right), \mu\right) \supseteq L^{p}\left(X, \Sigma_{1}(P), \mu\right)$. Choose $f \in$ $L^{2}\left(X, \Sigma_{\mathrm{d}}\left(P^{*}\right), \mu\right) \ominus L^{2}\left(X, \Sigma_{1}(P), \mu\right)$. By Theorem A on page 85 in [F] we have weak $\lim _{n \rightarrow \infty} P^{n} f=0$. On the other hand $P^{r n} f=f$ for every $n$. Therefore $f=0$. This proves that $\Sigma_{\mathrm{d}}\left(P^{*}\right)=\Sigma_{1}\left(P^{*}\right) \subseteq \Sigma_{\mathrm{d}}(P)$.

Now assume that $P$ a.o.s. and as before let $r_{X}$ stand for the length of the longest orthogonal sequence $\mathbf{1}_{A}, P \mathbf{1}_{A}, \ldots, P^{r_{X}-1} \mathbf{1}_{A}$, where $A \in \Sigma_{\mathrm{d}}\left(P^{*}\right)$. We have already noticed that $P^{r_{X}} \mathbf{1}_{A}=\mathbf{1}_{A}$ and $r_{X} \leq d$.

Suppose that $A$ is not an atom. Choose an arbitrary $\Sigma_{\mathrm{d}}\left(P^{*}\right) \ni B \varsubsetneqq A$. The functions $\mathbf{1}_{B}, P \mathbf{1}_{B}, \ldots, P^{r-1} \mathbf{1}_{B}$ are also pairwise orthogonal. If $P^{r_{X}} \mathbf{1}_{B}$ $=\mathbf{1}_{B}$ then the sequences $\mathbf{1}_{B}, P \mathbf{1}_{B}, \ldots, P^{r-1} \mathbf{1}_{B}, \ldots$ and $\mathbf{1}_{A \backslash B}, P \mathbf{1}_{A \backslash B}, \ldots$ $\ldots, P^{r-1} \mathbf{1}_{A \backslash B}, \ldots$ are disjoint. This contradicts the a.o.s. assumption. On the other hand if $P^{r_{X}} \mathbf{1}_{B} \neq \mathbf{1}_{B}$ we may produce a set $D=B \backslash B_{r_{X}}$ with $r_{D}>r_{X}$, contradicting the maximality of $r_{X}$. We conclude that $A$ is an atom. Because of a.o.s. we have $A \cup A_{1} \cup \ldots \cup A_{r_{X}-1}=X$. Clearly all $A_{j}$, where $0 \leq j \leq r-1$, are atoms as well. In particular $\Sigma_{\mathrm{d}}\left(P^{*}\right)$ is finite and atomic. We easily get $P^{n} \mathbf{1}_{B}=P^{s} \mathbf{1}_{B}$, where $s=n \bmod r$ and $r=r_{X}=R_{X}$ for simplicity.

The following corollary follows directly from Proposition 1 and Theorem $\mathrm{A}$ on page 85 in $[\mathrm{F}]$.

COROllary 1. A doubly stochastic operator $P$ with the a.o.s. property is weakly asymptotically cyclic. In particular for every $f \in L^{1}(X, \mathcal{A}, \mu)$ we have

$$
\text { weak } \lim _{n \rightarrow \infty}\left(P^{n} f-\beta \sum_{j=0}^{r-1}\left(\int_{A_{j}} f d \mu\right) \mathbf{1}_{A_{(j+n) \bmod r}}\right)=0,
$$

where $A_{0}, A_{1}, \ldots, A_{r-1}$ are the atoms of $\Sigma_{\mathrm{d}}\left(P^{*}\right)$ and $\beta=1 / \mu\left(A_{0}\right)$.

The next result is a generalization of Theorem 2 which was originally proved in [B3] using different methods. The present version has an "individual" character. In [B3] we assume that $\omega_{1}(f) \neq \emptyset$ for all $f \in \mathcal{D}$.

Theorem 1. Let $P$ be an a.o.s. doubly stochastic operator. If $f \in L^{1}(X$, $\mathcal{A}, \mu)$ is such that $\omega_{1}(f) \neq \emptyset$ then

$$
\lim _{n \rightarrow \infty}\left\|P^{n} f-\beta \sum_{j=0}^{r-1}\left(\int_{A_{j}} f d \mu\right) \mathbf{1}_{A_{(j+n) \bmod r}}\right\|_{1}=0,
$$

where $A_{0}, A_{1}, \ldots, A_{r-1}$ are the atoms of $\Sigma_{\mathrm{d}}\left(P^{*}\right)$ and $\beta=1 / \mu\left(A_{0}\right)$. 
Proof. Let $n_{k} \rightarrow \infty$ be such that $P^{n_{k}} f$ converges in $L^{1}$ norm to some $g$. Since $r$ is finite there exists a subsequence $n_{k_{j}}=$ const $=d \bmod r$. By Corollary 1 we have $g=\beta \sum_{j=0}^{r-1}\left(\int_{A_{j}} f d \mu\right) \mathbf{1}_{A_{(j+d) \bmod r}}$. Clearly $g$ is $P^{r}$-invariant. The convergence of $P^{r n} f$ to $g$ along some subsequence implies the convergence of the whole sequence $P^{r n} f$ as $P$ is a contraction. We get

$$
\lim _{n \rightarrow \infty}\left\|P^{r n+d} f-g\right\|_{1}=0 .
$$

After a slight reformulation we obtain the strong asymptotic cyclicity of $P^{n} f$ as all sequences $P^{n_{k}+j} f$ are norm convergent to $P^{j} g$.

The proof of Proposition 1 shows that $P^{r}=\operatorname{Id}$ on $L^{2}\left(X, \Sigma_{\mathrm{d}}\left(P^{*}\right), \mu\right)$ whenever $P$ i.a.o.s. However in this case $\Sigma_{\mathrm{d}}\left(P^{*}\right)$ is not necessarily finite (atomic). By $[\mathrm{F}]$, for $f \in L^{2}(X, \mathcal{A}, \mu)$, weak $\lim _{n \rightarrow \infty} P^{r n} f=E\left(f \mid \Sigma_{\mathrm{d}}\left(P^{*}\right)\right)$. In particular all weak limits of $P^{n} f$ are $P^{r}$-invariant. We obtain another generalization of [B3]:

Proposition 2. Let $P$ be an i.a.o.s. doubly stochastic operator. Then there exists $r \leq d$ ! such that for every $f \in L^{p}(X, \mathcal{A}, \mu)$ with $\omega_{p}(f) \neq \emptyset$, where $1 \leq p<\infty$, we have

$$
\lim _{n \rightarrow \infty}\left\|P^{r n} f-E\left(f \mid \Sigma_{\mathrm{d}}\left(P^{*}\right)\right)\right\|_{p}=0,
$$

where $E\left(\cdot \mid \Sigma_{\mathrm{d}}\left(P^{*}\right)\right)$ stands for the conditional expectation operator with respect to the $\sigma$-algebra $\Sigma_{\mathrm{d}}\left(P^{*}\right)$.

Proof. Without loss of generality we may confine our proof to $L^{2}(X, \mathcal{A}, \mu)$ only. As in the proof of Theorem 1 we show that $P^{r n} f$ converges in $L^{2}$ norm to some $g$. Given $f \in L^{2}(X, \mathcal{A}, \mu)$ let $f=f_{1}+f_{2}$, where $f_{1} \in$ $L^{2}\left(X, \Sigma_{\mathrm{d}}\left(P^{*}\right), \mu\right)$ and $f_{2} \perp L^{2}\left(X, \Sigma_{\mathrm{d}}\left(P^{*}\right), \mu\right)$. Since weak $\lim _{n \rightarrow \infty} P^{r n} f_{2}=$ 0 we have $g=f_{1}$ as $f_{1}$ is $P^{r}$-invariant. Clearly $f_{1}=E\left(f \mid \Sigma_{\mathrm{d}}\left(P^{*}\right)\right)$ and the proof is complete.

If $\Sigma_{\mathrm{d}}\left(P^{*}\right)$ is fully atomic (for instance when $P$ is Harris or simply kernel), then $X$ may be decomposed into disjoint cycles. Namely $X=$ $\bigcup_{k=1} \bigcup_{j=0}^{r_{k}-1} A_{k, j}$ and $P^{n} \mathbf{1}_{A_{k, j}}=\mathbf{1}_{A_{k,(j+n) \bmod r_{k}}}$. This in conjunction with Corollary 1 gives

COROllary 2. Let $P$ be an i.a.o.s. doubly stochastic operator on $L^{1}(X, \mathcal{A}, \mu)$. If $\Sigma_{\mathrm{d}}\left(P^{*}\right)$ is atomic with atoms $A_{k, j}$ described as above then for every $f$ we have

$$
\text { weak } \lim _{n \rightarrow \infty}\left(P^{n} f-\sum_{k=1} \sum_{j=0}^{r_{k}-1} \beta_{k}\left(\int_{A_{k, j}} f d \mu\right) \mathbf{1}_{A_{k,(j+n) \bmod r_{k}}}\right)=0,
$$

where $\beta_{k}=1 / \mu\left(A_{k, j}\right)$. If moreover $\omega_{1}(f) \neq \emptyset$ then the convergence $(2)$ is in norm. 
If $P$ is a Frobenius-Perron operator then a.o.s. implies strong asymptotic cyclicity because $P^{r}$ restricted to the spaces $L^{1}\left(A_{j}, \mathcal{A} \cap A_{j}, \mu\left(\cdot \cap A_{j}\right)\right)$ is asymptotically stable. This is because the tail $\sigma$-algebras of all $\left.P^{r}\right|_{A_{j}}$ are trivial. The same result is obtained in $[\mathrm{BB}]$ using functional arguments. We recall that $\lim _{n \rightarrow \infty} P^{* n} P^{n}=Q$ exists in the $L^{2}$ strong operator topology. Obviously $Q$ is doubly stochastic. It has been noticed in [BB] that $Q$ is a projection $\left(Q^{2}=Q\right)$ if $P$ is Frobenius-Perron. Clearly $Q$ is a projection if $P$ is strongly asymptotically cyclic. Here we adapt some elements of [BB] to show:

TheOrem 2. Let $P$ be an a.o.s. doubly stochastic operator on $L^{1}(X, \mathcal{A}, \mu)$. Then the following conditions are equivalent:

(i) $P$ is strongly asymptotically cyclic,

(ii) $Q$ and $P$ commute,

(iii) $Q$ is a projection.

Proof. (i) $\Rightarrow$ (ii). Let $f \in L^{2}(\mu)$. It follows from Theorem 1 that

$$
\lim _{n \rightarrow \infty} P^{r n} f=\sum_{j=0}^{r-1} \frac{1}{\mu\left(A_{j}\right)}\left(\int_{A_{j}} f d \mu\right) \mathbf{1}_{A_{j}}=E(f)
$$

in $L^{2}$ norm (we may switch from $L^{1}$ to $L^{2}$ because all $L^{p}$ strong operator topologies, where $1 \leq p<\infty$, coincide on the set of doubly stochastic operators; see $[\mathrm{Br}]$ for the details). Since $P^{* r} P^{r}=\operatorname{Id}$ on $L^{2}\left(X, \Sigma_{\mathrm{d}}\left(P^{*}\right), \mu\right)$ and $P^{*}$ is an $L^{2}$ contraction we get

$$
P^{* r} E(f)=P^{* r} \lim _{n \rightarrow \infty} P^{r n} f=P^{* r} P^{r} \lim _{n \rightarrow \infty} P^{r(n-1)} f=E(f) .
$$

We have

$$
\begin{aligned}
\|Q f-E(f)\|_{2} & =\lim _{n \rightarrow \infty}\left\|P^{* r n} P^{r n} f-E(f)\right\|_{2} \\
& =\lim _{n \rightarrow \infty}\left\|P^{* r n}\left(P^{r n} f-E(f)\right)\right\|_{2} \\
& \leq \lim _{n \rightarrow \infty}\left\|P^{r n} f-E(f)\right\|_{2}=0 .
\end{aligned}
$$

This means that $Q=E$. Now (ii) is clear as

$$
Q P f=E(P f)=\lim _{n \rightarrow \infty} P^{r n} P f=P \lim _{n \rightarrow \infty} P^{r n} f=P E(f)=P Q f .
$$

(ii) $\Rightarrow$ (iii). For every $n$ and $f \in L^{2}(\mu)$ we have $Q f=P^{* n} Q P^{n} f$. If $Q$ and $P$ commute then

$$
Q f=P^{* n} Q P^{n} f=P^{* n} P^{n} Q f=\lim _{n \rightarrow \infty} P^{* n} P^{n} Q f=Q^{2} f .
$$

Therefore $Q$ is a projection.

(iii) $\Rightarrow$ (i). It follows from Proposition 1 that $P$ is weakly asymptotically cyclic. First we note that the invariant $\sigma$-algebra $\Sigma_{\mathrm{i}}(Q)$ coincides with $\Sigma_{\mathrm{d}}\left(P^{*}\right)$. This easily follows from the identity $Q=P^{* n} Q P^{n}$. In fact, given 
$A \in \Sigma_{\mathrm{i}}(Q)$ we apply Lemma 0 from [KL] to obtain $P^{n} \mathbf{1}_{A}=\mathbf{1}_{A_{n}}$ for every natural $n$. This gives $A \in \Sigma_{\mathrm{d}}\left(P^{*}\right)$. On the other hand if $P^{n} \mathbf{1}_{A}=\mathbf{1}_{A_{n}}$ then obviously $P^{* n} P^{n} \mathbf{1}_{A}=\mathbf{1}_{A}$ and passing with $n$ to infinity we obtain $A \in \Sigma_{\mathrm{i}}(Q)$. The equality $\Sigma_{\mathrm{i}}(Q)=\Sigma_{\mathrm{d}}\left(P^{*}\right)=\Sigma_{1}(P)$ is proved. We get

$$
Q f=E\left(f \mid \Sigma_{\mathrm{d}}\left(P^{*}\right)\right)=\sum_{j=0}^{r-1} \frac{1}{\mu\left(A_{j}\right)}\left(\int_{A_{j}} f d \mu\right) \mathbf{1}_{A_{j}},
$$

where $A_{0}, A_{1}, \ldots, A_{r-1}$ are the atoms of $\Sigma_{\mathrm{d}}\left(P^{*}\right)$. In particular we have $Q f=\left(1 / \mu\left(A_{j}\right)\right)\left(\int f d \mu\right) \mathbf{1}_{A_{j}}$ if $f$ is concentrated on $A_{j}$. Repeating arguments from [BB] for every $f \in \mathcal{D}$ which is concentrated on $A_{j}$ we get

$$
\begin{aligned}
\left\|P^{r n} f-\frac{1}{\mu\left(A_{j}\right)} \mathbf{1}_{A_{j}}\right\|_{2}^{2} & =\int\left(P^{r n} f-\frac{1}{\mu\left(A_{j}\right)} \mathbf{1}_{A_{j}}\right)\left(P^{r n} f-\frac{1}{\mu\left(A_{j}\right)} \mathbf{1}_{A_{j}}\right) d \mu \\
& =\int P^{r n} f \cdot P^{r n} f d \mu-\frac{1}{\mu\left(A_{j}\right)} \\
& =\int P^{* r n} P^{r n} f \cdot f d \mu-\frac{1}{\mu\left(A_{j}\right)} \rightarrow \int Q f \cdot f d \mu-\frac{1}{\mu\left(A_{j}\right)} \\
& =\int \frac{1}{\mu\left(A_{j}\right)} \cdot f d \mu-\frac{1}{\mu\left(A_{j}\right)}=0 .
\end{aligned}
$$

Since $A_{j}$ 's cover the whole space $X$ we obtain

$$
\lim _{n \rightarrow \infty}\left\|P^{r n} f-\sum_{j=0}^{r-1}\left(\int_{A_{j}} f d \mu\right) \frac{1}{\mu\left(A_{j}\right)} \mathbf{1}_{A_{j}}\right\|_{2}=0
$$

for every $f \in L^{2}(\mu)$. Clearly the convergence $P^{r n} f \rightarrow E(f)$ in $L^{2}(\mu)$ implies the norm convergence in $L^{1}(\mu)$, thus $P_{S}$ is strongly asymptotically cyclic.

FinAL REMARKS. It is not generally true that a doubly stochastic operator which overlaps supports is asymptotically stable. A suitable counterexample was supplied by R. Rudnicki and may be found in [R2].

Let $P_{S}$ be a Frobenius-Perron operator with stationary density $f_{*}$. If $P_{S}$ a.o.s. then it is strongly asymptotically cyclic even if $\operatorname{supp}\left(f_{*}\right) \neq X$. This was proved by R. Zaharopol [Z]. Roughly speaking this is because $\bigcup_{n=1}^{\infty} S^{-n}\left(\operatorname{supp}\left(f_{*}\right)\right)=X$, which easily follows from a.o.s.

On the other hand there are kernel Markov operators $P$ with stationary densities and overlapping supports which are not asymptotically stable. For this consider $X=\mathbb{N} \cup\{0\}$ with counting measure $\mu$, and let

$$
p_{i, j}= \begin{cases}1 & \text { if } i=j=0, \\ 1 / 2^{i} & \text { if } j=0 \text { and } i \neq 0, \\ 1-1 / 2^{i} & \text { if } j=i+1 \text { and } i \neq 0,\end{cases}
$$

be transition probabilities. In the standard way the matrix $\left[p_{i, j}\right]$ defines a 
Markov operator (chain) on $\ell^{1}(X)$. Namely we set $\operatorname{Pf}(j)=\sum_{i=0}^{\infty} f(i) p_{i, j}$. Clearly $P$ overlaps supports as $P f(0)>0$ for any nonnegative nonzero $f$, and $f_{*}=\delta_{0}$ is the only stationary density. On the other hand we have $\lim _{n \rightarrow \infty} \int_{\{0\}} P^{n} f d \mu<1$ for every $f \in \mathcal{D}$ which is not entirely concentrated on $\{0\}$. Hence $P$ is not asymptotically stable.

\section{References}

[B1] W. Bartoszek, Asymptotic stability of iterates of positive contractions on Banach lattices, in: Proc. Int. Conf. Function Spaces (Poznań, 1986), Teubner Texte zur Math. 103, Teubner, 1986, 153-157.

[B2] - Asymptotic periodicity of the iterates of positive contractions on Banach lattices, Studia Math. 91 (1988), 179-188.

[B3] - Asymptotic properties of the iterates of stochastic operators on (AL) Banach lattices, Ann. Polon. Math. 52 (1990), 165-173.

[BB] W. Bartoszek and T. Brown, On Frobenius-Perron operators which overlap supports, Bull. Polish Acad. Sci. Math. 45 (1997), 17-24.

[Br] J. R. Brown, Ergodic Theory and Topological Dynamics, Academic Press, New York, 1976.

[F] S. R. Foguel, The Ergodic Theory of Markov Processes, Van Nostrand Reinhold, New York, 1969.

[K1] J. Komornik, Asymptotic periodicity of the iterates of Markov operators, Tôhoku Math. J. 38 (1986), 15-27.

[K2] - Asymptotic decomposition of Markov operators, Bull. Polish Acad. Sci. Math. 35 (1987), 321-327.

[KL] U. Krengel and M. Lin, On the deterministic and asymptotic $\sigma$-algebras of a Markov operator, Canad. Math. Bull. 32 (1989), 64-73.

[L] A. Lasota, Invariant principle for discrete time dynamical systems, Univ. Iagel. Acta Math. 31 (1994), 111-127.

[LM] A. Lasota and M. C. Mackey, Chaos, Fractals and Noise: Stochastic Aspects of Dynamics, Springer, New York, 1993.

[R1] R. Rudnicki, On asymptotic stability and sweeping for Markov operators, Bull. Polish Acad. Sci. Math. 43 (1995), 245-262.

[R2] - , Asymptotic stability of Markov operators: a counter-example, ibid. 45 (1997), $1-5$.

[Z] R. Zaharapol, Strongly asymptotically stable Frobenius-Perron operators, preprint, 1997.

Department of Mathematics

University of South Africa

P.O. Box 392

Pretoria 0003, South Africa

E-mail: bartowk@alpha.unisa.ac.za 